\title{
Tecnologia educacional híbrida- blended learning no olhar do discente de
}

\section{enfermagem}

\author{
Hybrid education technology- blended learning in the look of the nursing student \\ Tecnologia educacativa híbrida learning mesclado en el aspecto del estudiante de enfermeria
}

Recebido: 14/03/2021 | Revisado: 20/03/2021 | Aceito: 30/03/2021 | Publicado: 10/04/2021

Najara Araújo da Silva

ORCID: https://orcid.org/0000-0002-4896-4867 Centro Universitário Anhanguera, Brasil

E-mail: najara27sp@hotmail.com

Larissa Leocadio da Silva

ORCID: https://orcid.org/0000-0003-3828-0792 Centro Universitário Anhanguera, Brasil

E-mail: larissaleocadio.s@gmail.com

Daylene dos Santos Souza

ORCID: https://orcid.org/0000-0002-6723-6099 Centro Universitário Anhanguera, Brasil E-mail: daylenesouza98@hotmail.com

Jaqueline Santos Viana

ORCID: https://orcid.org/0000-0002-5734-191X Centro Universitário Anhanguera, Brasil E-mail: jaqueline.viana@anhanguera.com

Jefferson Carlos de Oliveira

ORCID: https://orcid.org/0000-0002-5258-7099 Centro Universitário Anhanguera, Brasil

E-mail: jefferson.c.oliveira@ anhanguera.com

\begin{abstract}
Resumo
Objetivo: O estudo em questão teve como objetivo identificar a fluência digital por meio da utilização do questionário, de Piconez nos graduandos de enfermagem. Método: Estudo de abordagem transversal, quantitativo, composto por 91 graduandos distribuídos entre o $1^{\circ}$ ao $5^{\circ}$ semestre do curso de Enfermagem. A coleta de dados ocorreu no mês de abril de 2019. Aplicado um formulário contendo os dados sociodemográficos e o Instrumento de Piconez. Os dados foram apresentados de modo descritivo. O projeto foi submetido a Plataforma Brasil sob o ${ }^{\circ}$ CAEE 89610218.1.0000.5493. Resultado: A variável predominante foi $68 \%$ gênero feminino e $23 \%$ gênero masculino. Quanto analisado o semestre, $24 \%$ eram do $5^{\circ}$ semestre, $22 \%$ do $1^{\circ}$ semestre, $17 \%$ do $2^{\circ}$ e $4^{\circ}$ semestre, $10 \%$ do $3{ }^{\circ}$ semestre e $1 \%$ nulo. Conclusão: $\mathrm{O}$ uso da tecnologia na modalidade hibrida abre precedentes e lacunas a serem exploradas e mobilizadas para o uso como estratégias de ensino. Nessa perspectiva traz inovações a serem utilizadas e implementadas no que tange a abordagem pedagógica como mediador do conhecimento e aprendizagem.

Palavras-chave: Tecnologia educacional; Educação em saúde; Enfermagem; Ensino.
\end{abstract}

\begin{abstract}
Objective: The study in question aimed to identify digital fluency through the use of the questionnaire, from Piconez in nursing undergraduates. Method: Study of cross-sectional, quantitative approach, composed of 91 undergraduate students distributed between the 1st to the 5th semester of the nursing course. Data were collected in April 2019. A form containing the sociodemographic data and the Piconez Instrument was applied. The data were presented descriptively. The project was submitted to Plataforma Brasil under the number CAEE 89610218.1.0000.5493. Result: The predominant variable was $68 \%$ female and $23 \%$ male. When analyzed in the semester, $24 \%$ were from the 5th semester, $22 \%$ from the 1 st semester, $17 \%$ from the 2 nd and 4th semester, $10 \%$ from the 3 rd semester and $1 \%$ null. Conclusion: The use of technology in the hybrid modality opens precedents and gaps to be explored and mobilized for use as teaching strategies. In this perspective, it brings innovations to be used and implemented with regard to the pedagogical approach as a mediator of knowledge and learning.
\end{abstract}

Keywords: Educational technology; Health education; Nursing; Teaching.

\section{Resumen}

Objetivo: El estudio en cuestión tenía como objetivo identificar la fluidez digital a través del uso del cuestionario, de Piconez en los estudiantes de enfermería. Método: Estudio del enfoque transversal y cuantitativo, compuesto por 91 estudiantes de grado distribuidos entre el $1^{\circ}$ y el $5^{\circ}$ semestre del curso de enfermería. Los datos se recopilaron en abril 
de 2019. Se aplicó un formulario que contenía los datos sociodemográficos y el Instrumento Piconez. Los datos se presentaron descriptivamente. El proyecto fue presentado a Plataforma Brasil bajo el número CAEE 89610218.1.0000.5493. Resultado: La variable predominante fue $68 \%$ femenina y $23 \%$ masculina. Cuando se analizaron en el semestre, el $24 \%$ eran del quinto semestre, el $22 \%$ del primer semestre, el $17 \%$ del segundo y cuarto semestre, el 10\% del tercer semestre y el 1\% nulos. Conclusión: El uso de la tecnología en la modalidad híbrida abre precedentes y lagunas que deben explorarse y movilizarse para su uso como estrategias de enseñanza. Desde esta perspectiva, aporta innovaciones para ser utilizadas e implementadas con respecto al enfoque pedagógico como mediador del conocimiento y el aprendizaje.

Palabras clave: Tecnología educativa; Educación para la Salud; Enfermería; Ensenãnza.

\section{Introdução}

A educação em saúde apresenta papel fundamental no que tange as práticas de promoção da saúde e, de seus princípios como uma estratégia a romper barreiras tecnicistas (Câmara, 2012). Assim, a educação em saúde no Brasil surgiu com base em condições e situações emergentes, políticas e econômicas que contribuíram para elevar reflexões e mudanças de como interagir entre o profissional de saúde e o cliente buscando remeter as questões de promoção e prevenção em saúde (Sousa et al, 2018).

A Enfermagem, neste contexto, presenciou e contextualizou todo esse processo de transformação da educação tornando influentes as inserções do mesmo na sua prática (Sousa et al, 2018).

As ações educacionais em grupos consentem uma opção importante na busca de promoção da saúde, permitindo o aprofundamento de altercações e o acréscimo de conhecimento, para que as pessoas superem as suas dificuldades e alcancem maior autonomia, melhores qualidades de saúde e qualidade de vida (Coelho, 2009).

Nesse contexto, Coelho (2009), afirma que as tecnologias educativas em saúde (TESs) são estratégias expressivas que culminam para desenvolver o trabalho educativo e o processo do cuidar. Essas estratégias agregam o grupo das tecnologias leves, designadas tecnologias de relações, como acolhimento, o vínculo, automação, responsabilização e gestão como modo de gerir os métodos de trabalho.

No contexto educacional o uso de novas tecnologias, tem o professor como protagonista da conexão entre o ensino e a aprendizagem, pois não há especificamente uma única maneira de se utilizar os recursos e oportunidades educativas especificas entre o aluno e a escola. É necessário realizar as adaptações que contribuam para que o professor tenha domínio quanto ao uso da tecnologia na mediação do processo de educação (Inocêncio, 2016).

A utilização da tecnologia educativa nos últimos anos tem sido explorada no sentido de ser uma didática utilizada como metodologia no ensino-aprendizagem beneficiando com isso aptidões individuais e, propiciando desempenhos adequados no contexto do aprendizado (Maia, 2018).

Atualmente, a educação dispõe indivíduos para o Sistema de informação, porque já não se aprende apenas no ato de ensinar, ou seja, as pessoas aprendem também pelo autodidatismo, pelos colegas e amigos através da mídia e Internet.

Nesse contexto o ensino também não se reduz ao que se planeja intencionalmente, ou seja, o ensino hibrido associa processos abertos e informais. A maneira de aprender poderá advir quando estamos sozinhos ou juntos aos docentes, podendo acontecer espontâneo ou intencional (Jiménez, 2019).

Portanto, não existe um único modo de aprender, existem formas diferentes podendo o trabalho colaborativo aliar-se ao uso de tecnologias digitais e propiciar o aprendizado e troca de barreiras além de uma sala de aula. O termo blended learning surge em 2001, com a definição de misturar o melhor da educação online, com o melhor da educação presencial, construindo assim o modelo hibrido (Silva, 2016).

Na conjuntura contemporânea cada vez mais há meios excepcionais de informações que circulam e são produzidas na educação. Os conhecimentos de fundem tornando-se um ciclo de renovação assimilados por grande parte das pessoas. Nesse 
cenário a tecnologia digital como suporte facilitador abre precedente a fim de desenvolver o ensino e a aprendizagem, e aborda características conceituais e estruturais que devem ser melhor compreendidas e contextualizadas na pratica educacional.

Segundo Nassif (2019), o termo hibrido pode ser conceituado como uma mistura, e "blended" uma mescla a qual combina a educação por meios de diferentes tempos, espaços, atividades metodológicas com conectividade e mobilidade mais perceptível, profunda e ampla.

Pode-se adotar uma proposta de ensino mediante o contexto a ser trabalhado para que ocorra a intensificação e a troca de experiências. Podemos definir a educação on-line como um conjunto de ações permeadas e desenvolvidas de meios de aprendizagem, métodos e dispositivos de comunicação além de meios informacionais, com o uso da internet.

Assim, torna-se extremamente desafiador a área da enfermagem diante dos avanços tecnológicos de informação e comunicação entre os acadêmicos (Nassif, 2019). Podemos destacar a utilização das tecnologias de informação e comunicação em ambientes virtuais de enfermagem, sendo uma promissora e atraente ferramenta para o favorecimento da construção de aprendizagem pedagógica (Costa, 2011).

Mediante a necessidade da inserção de propostas educacionais o ambiente virtual de aprendizagem visa fomentar o ensino interativo e flexível podendo ser aplicado a enfermagem. Portanto, para que seja efetivo sua utilização torna-se viável o reconhecimento do perfil dos alunos e suas condições para que se possa implementar medidas e propostas adequadas. Portanto, o estudo em questão tem como finalidade testar a utilização do questionário, de Piconez que possibilita avaliar o conhecimento digital tecnológico (Costa, 2011).

Desse modo, levando em consideração que a tecnologia poderá propiciar ferramentas educacionais no contexto educacional e suas áreas correlatas possibilidades para que os alunos interajam, discutam, exercitem habilidades distintas, reflitam sobre elas, a partir de um espaço que proporcione maior liberdade e imersão, esta pesquisa teve por objetivo testar a utilização do questionário de Piconez para avaliar o conhecimento digital tecnológico de alunos do curso de Enfermagem de uma Universidade Privada.

\section{Metodologia}

O delineamento do estudo é de abordagem transversal, quantitativo, composto por 91 graduandos distribuídos entre o $1^{\circ}$ ao $5^{\circ}$ semestre do curso de graduação em Enfermagem (Pereira, 2018).

A coleta de dados ocorreu no mês de abril de 2019. Os critérios de inclusão para esta pesquisa foram os seguintes: Ser aluno de graduação em enfermagem e apresentarem noções básicas sobre o uso da tecnologia (Word, Excel, Power Point e uso da tecnologia digital); serão considerados excluídos os alunos que não assinarem o TCLE ou não estarem presentes durante a aplicação dos instrumentos e a coleta de dados.

O estudo foi realizado no Centro Universitário Anhanguera - Vila Mariana na cidade de São Paulo. Trata-se de um Centro Universitário privado do município de São Paulo, destacando-se pelas atividades de ensino e pesquisa.

Os alunos foram convidados e orientados quanto à finalidade do estudo e, após a sua aquiescência, assinaram o Termo de Consentimento Livre e Esclarecido (TCLE), de acordo com as Normas Éticas Internacionais para pesquisa em seres humanos seguindo os preceitos da resolução CNS 466/12 (BRASIL, 2013).

Foi aplicado formulário contendo os dados sociodemograficos (sexo, faixa etária, etnia, religião, renda familiar, escolaridade e procedência) e na sequencia foi aplicado questionário de Piconez (2009) que possibilita avaliar o conhecimento digital tecnológico.

A coleta de dados ocorreu em uma sala privativa de modo que fosse garantido o anonimato e a privacidade do aluno. Os dados foram coletados em local privativo no próprio centro universitário cedido pela coordenação do curso. O tempo 
estimado para a coleta de dados para cada aluno foi em média de 30 minutos. Os dados foram apresentados mediante a estatística descritiva por meio de tabelas, quadros, contendo números absolutos e percentuais. Para esta pesquisa foi atribuído o risco mínimo sendo facultada ao sujeito da pesquisa a retirada da anuência do TCLE a qualquer momento do estudo sem acarretar qualquer prejuízo ou danos morais aos mesmos. O estudo em questão foi submetido ao Comitê de Ética Anhanguera de São Paulo (UNIAN) e submetido a Plataforma Brasil sob o n CAEE 89610218.1.0000.5493, conforme a Resolução CNS no $466 / 12$ (Brasil, 2013).

\section{Resultados e Discussão}

A partir de da amostra de 91 graduandos de enfermagem os dados sócio demográficos serão apresentados na Tabela 1.

Tabela 1. Caracterização das variáveis sócio demográficas dos graduandos de enfermagem - São Paulo, 2021.

\begin{tabular}{lll}
\hline VARIÁVEIS & Grupo Híbrido \\
& N & \% \\
\hline GÊNERO & & \\
Masculino & 23 & 25,3 \\
Feminino & 68 & 74,7 \\
Subtotal & $\mathbf{9 1}$ & $\mathbf{1 0 0}$ \\
RAÇA & & \\
Branco & 33 & 36,2 \\
Pardo & 31 & 34,0 \\
Negro & 20 & 22,2 \\
Amarelo & 02 & 2,1 \\
Nulo & 05 & 5,4 \\
Subtotal & $\mathbf{9 1}$ & $\mathbf{1 0 0}$ \\
RELIGIÃO & & \\
Católico & 28 & 30,8 \\
Evangélico & 26 & 28,5 \\
Espírita & 10 & 11,0 \\
Outros & 27 & 29,7 \\
Subtotal & $\mathbf{9 1}$ & $\mathbf{1 0 0}$ \\
\hline
\end{tabular}

Fonte: Autores (2021).

Os dados da Tabela 1, demonstram que $68 \%$ da amostra do estudo foi composta pelo gênero feminino, $33 \%$ brancos, quanto a religião $31 \%$ eram católicos e $31 \%$ evangélicos. Esta relação é percebida mediante a faixa o gênero feminino ainda ser predominante no curso de graduação em enfermagem. 
Tabela 2. Caracterização das variáveis escolaridade e semestre dos graduandos de enfermagem - São Paulo, 2021.

\begin{tabular}{lll} 
VARIÁVEIS & Grupo Híbrido \\
& $\mathbf{N}$ & $\mathbf{\%}$ \\
\hline GRAU DE ESCOLARIDADE & & \\
Médio Completo & 14 & 15,3 \\
Superior Completo & 1 & 1,1 \\
Superior Incompleto & 76 & 83,6 \\
Subtotal & $\mathbf{9 1}$ & $\mathbf{1 0 0}$ \\
SEMESTRE & & \\
$1^{\circ}$ & 22 & 24,2 \\
$2^{\circ}$ & 17 & 19,7 \\
$3^{\circ}$ & 10 & 11,0 \\
$4^{\circ}$ & 17 & 18,7 \\
$5^{\circ}$ & 24 & 26,4 \\
\hline Total & $\mathbf{9 1}$ & $\mathbf{1 0 0}$ \\
\hline
\end{tabular}

Fonte: Autores (2021).

Em relação a escolaridade prevaleceu alunos com superior incompleto $76 \%$, quando analisado o semestre obteve-se $24 \%$ que cursavam o $5^{\circ}$ semestre. Quanto ao semestre das turmas espera-se que o aluno tenha desenvolvido maior habilidades quanto ao manuseio das ferramentas digitais para a utilização em seus estudos e trabalhos acadêmicos.

Tabela 3. Auto avaliação do conhecimento e da habilidade técnica em informática com aplicação do instrumento de Piconez, dos alunos de graduação em enfermagem- São Paulo, 2021.

\begin{tabular}{|c|c|c|c|c|}
\hline \multirow[t]{2}{*}{ Conhecimento e habilidade técnica em informática } & \multicolumn{2}{|c|}{ Sim } & \multicolumn{2}{|c|}{ Não } \\
\hline & $N$ & $\%$ & $\mathrm{~N}$ & $\%$ \\
\hline Sou capaz de instalar um programa em meu computador? & 65 & 71,4 & 26 & 28,5 \\
\hline $\begin{array}{l}\text { Sou capaz de criar uma pasta em minha } \\
\text { área de trabalho ou arquivo de documentos }\end{array}$ & 84 & 92,3 & 7 & 7,6 \\
\hline Sou capaz de copiar e mover um arquivo para uma pasta? & 86 & 94,5 & 5 & 5,4 \\
\hline Sou capaz de criar um atalho usando o Windows? & 71 & 78,0 & 20 & 21,9 \\
\hline Sei configurar o relógio do meu computador? & 81 & 89,0 & 10 & 10,9 \\
\hline $\begin{array}{l}\text { Sei onde encontrar informação documentada caso não } \\
\text { Entenda alguma parte de um programa? }\end{array}$ & 65 & 71,4 & 26 & 28,5 \\
\hline $\begin{array}{l}\text { Sei como mover arquivos indesejados para a lixeira e } \\
\text { Remove-los definitivamente quando não quero? }\end{array}$ & 85 & 93,4 & 6 & 6,5 \\
\hline Sou capaz de descompactar um arquivo compactado? & 47 & 51,6 & 44 & 48,3 \\
\hline Sei o quanto fazer regularmente uma cópia (backup) & 77 & 84,6 & 14 & 15,3 \\
\hline De arquivos importantes? & & & & \\
\hline Já obtive sucesso em comprar um computador? & 72 & 79,1 & 19 & 20,8 \\
\hline Sei como participar de grupos de discussão on-line? & 42 & 46,1 & 48 & 52,7 \\
\hline Sei o que é um plug-in e como instalá-lo? & 37 & 40,6 & 53 & 58,2 \\
\hline Total & 100 & & 100 & \\
\hline
\end{tabular}

Fonte: Autores (2021). 
Os dados apresentados acima descrevem que $84 \%$ dos alunos são capazes de criar uma pasta em sua área de trabalho, 86\% são capazes de copiar e mover arquivos para a pasta, $71 \%$ é capaz de criar um atalho usando o Windows, $81 \%$ sabem como configurar o relógio do computador, $65 \%$ sabem onde encontrar informações documentadas caso não encontrem alguma parte de um programa, $85 \%$ dos participantes sabem como mover arquivos indesejáveis para o lixo, $47 \%$ dos alunos sabem como descompactar um arquivo, $77 \%$ sabem realizar uma cópia do backup, $72 \%$ obtiveram sucesso na compra de um computador e 48\% relatam não obter sucesso em participar de grupos de discussão on-line. Esses resultados revelaram que os alunos apresentaram um bom desempenho quanto analisados pelo instrumento de Piconez, exceto a participação em grupos de discussão on-line.

No que diz respeito à fluência digital, nota-se que os alunos apresentaram conhecimento e habilidades quanto ao uso da tecnologia como forma de aprendizagem sendo fluentes digitais, visto que são capazes de encontrar, utilizar de modo eficaz, eficiente as informações que necessitam. Desse modo, metodologias de ensino mediadas pela tecnologia são extremamente relevantes para contribuir e diversificar formas e maneiras de comunicação entre professores e alunos (Peres, 2004).

Assim, a tecnologia e a ciência tem alcançados e provocados profundas mudanças e efeitos no que tange a educação do ensino superior. Estas mudanças acontecem de modo avassalador, pelas tecnologias de informação sendo permeadas de maneira geral pela sociedade, o meio acadêmico, proporcionando mudanças em processos e transformando sujeitos em protagonistas (Fernandes, 2015). Cabe destacar e refletir que a utilização de recursos e propostas que abarquem conteúdos pedagógicos mediados pro recursos tecnológicos serão instrumentos de diálogo e interação em formas de saberes abrindo precedentes para o processo de aprendizagem rompendo paradigmas e o modelo tradicional (Moraes, 2015).

No que diz respeito a utilização de ferramentas tais como o fórum e o bate-papo, disponíveis em ambiente virtual de aprendizagem em atividade com estudantes de enfermagem foi demostrada uma baixa interação. No entanto, deve-se promover ao aluno condições para a construção do conhecimento, de modo que seja mediado pela tecnologia e a presença do professor, favorecendo e consolidando as etapas deste processo (Miranda, 2020).

Deve-se contextualizar as relações e estruturas escolares onde o professor seja colocado em um cenário alicerçado de mudanças e práticas pedagógicas que assumam um caráter desafiador diferenciando-se de métodos convencionais, esse novo contexto abre lacunas para o modelo hibrido envolvido pelas tecnologias, permeado pela importância do professor modificando sua atuação enquanto docente (Donato, 2018).

Os resultados deste estudo superam dados descritos em uma pesquisa realizada com 80 alunos de uma escola pública de enfermagem realizada em 2003, cabe destacar que o intervalo de tempo entre o estudo é um ponto significativo para o avanço na incorporação de tecnologias e comunicação entre universitários (Almeida, 2003).

Nessa priori, deve-se elencar os benefícios e o modo consciente de riscos, pois todos benefícios também são atrelados a riscos, sendo crucial a manutenção da criticidade para que ocorram avanços tecnológicos e que sejam reconhecidos os benefícios e riscos advindos, levando o aluno ao desenvolvimento e ao amadurecimento de inserção de novas propostas a serem incorporadas em seu processo de aprendizagem permeado pelas tecnologias.

\section{Conclusão}

Por meio dos resultados, foi possível concluir que os alunos do curso de graduação em Enfermagem do Centro Universitário da Anhanguera da Vila Mariana de São Paulo foram considerados como fluentes digitais, possuindo conhecimento, habilidade no uso de ambientes virtuais de aprendizagem em sua formação acadêmica. Torna-se viável como sendo uma ferramenta a ser aperfeiçoada a pratica pedagógica pelos docentes a fim de realizar transformações que re- 
signifiquem saberes e práticas e concepções advindas com o uso de tecnologias digitais. Portanto, torna-se primordial novas pesquisas acerca da temática e suas ponderações sobre o ensino hibrido.

\section{Agradecimentos}

Artigo oriundo do projeto intitulado: "Tecnologia educacional hibrida- blended learning no olhar do discente de enfermagem" financiado pela FUNADESP- Fundação Nacional de Desenvolvimento do Ensino Superior Particular.

\section{Referências}

Almeida, M. E. B. (2003). Educação a distância na internet: abordagens e contribuições dos ambientes digitais de aprendizagem. Educação e Pesquisa, 29(2), 327-340. https://doi.org/10.1590/S1517-97022003000200010

BRASIL. Conselho Nacional de Saúde.Resolução no 466, de 12 de dezembro de 2012. Aprova diretrizes e normas regulamentadoras de pesquisas envolvendo seres humanos. Diário Oficial [da] República Federativa do Brasil. 59. <http://conselho.saude.gov.br/resolucoes/2012/ Reso466.pdf>.

Câmara, A. M. C. S., Melo, V. L. C., G., M. G. P., Pena, B. C., Silva, A. P. Oliveira, K. M., Moraes, A. P. S., Coelho, G. R., \& Victorino, L. R. (2012). Percepção do processo saúde-doença: significados e valores da educação em saúde. Revista Brasileira de Educação Médica,36(1), 4050. https://doi.org/10.1590/S0100-55022012000200006

Coelho, M. O., \& Jorge, M. S. B. (2009). Tecnologia das relações como dispositivo do atendimento humanizado na atenção básica à saúde na perspectiva do acesso, do acolhimento e do vínculo. Ciência \& Saúde Coletiva, 14(1), 1523-1531. https://doi.org/10.1590/S1413-81232009000800026

Costa, P. B., Prado, C., Oliveira, L. F. T., Peres, H. H. C., Massarollo, M. C. K. Braga, F., M.de F. P., Leite, M. M. J., \& Freitas, G. F. (2011). Fluência digital e uso de ambientes virtuais: caracterização de alunos de enfermagem. Revista da Escola de Enfermagem da USP, 45, 15891594. https://dx.doi.org/10.1590/S0080-62342011000700008

Donato S., J., \& José B., I. (2018). Blended learning: o ensino híbrido e a avaliação da aprendizagem no ensino superior. Boletim Técnico Do Senac, 44(1). https://doi.org/10.26849/bts.v44i1.648

Fernandes, V., \& Frison, L. (2015). Estratégias de aprendizagem autorregulatória no ensino superior: escrita de um artigo científico. Psicologia da Educação, O(41), 37-49. https://revistas.pucsp.br/index.php/psicoeduca/article/view/27841

Inocêncio, A. C. G., \& Ribeiro, M. W. de S. (2016). O uso de "tecnologias" no processo de ensino-aprendizagem. Itinerarius Reflectionis, 12(1). https://doi.org/10.5216/rir.v12i1.37151

Jiménez-Saiz, R., \& Rosace, D. (2019). Is hybrid-PBL advancing teaching in biomedicine? A systematic review. BMC medical education, $19(1), 226$. https://doi.org/10.1186/s12909-019-1673-0

Maia, J. D. de S., Silva, A. B., Melo, R. H. V., Rodrigues, M. P., \& Junior, A. M. (2018). A educação em saúde para usuários hipertensos: percepções de profissionais da estratégia saúde da família. Revista Ciência Plural,4(1), 81-97. https://periodicos.ufrn.br/rcp/article/view/13634

Miranda, R. V., Moret, A. de S., e Silva, J. C., \& Perpetua Simão, B. (2020). Ensino Híbrido: Novas Habilidades Docentes Mediadas pelos Recursos Tecnológicos. EaD Em Foco, 10(1). https://doi.org/10.18264/eadf.v10i1.913

Moraes, R. de A. (2015). Educação, trabalho e novas tecnologias na educação a distância: uma reflexão crítica. Revista HISTEDBR On-Line, 15(65), 103-112. https://doi.org/10.20396/rho.v15i65.8642699

Nassif, J., Sleiman, A. K., Nassar, A. H., Naamani, S., \& Sharara-Chami, R. (2019). Hybrid Simulation in Teaching Clinical Breast Examination to Medical Students. Journal of cancer education : the official journal of the American Association for Cancer Education,34(1), 194-200. https://doi.org/10.1007/s13187-017-1287-3

Neves, C. A. B. (2008). Saúde: a cartografia do trabalho vivo. Cadernos de Saúde Pública, 24(8), 1953-1955. https://doi.org/10.1590/S0102$311 \mathrm{X} 2008000800023$

Pereira A. S. et al. (2018). Metodologia da pesquisa científica. UFSM. https://repositorio.ufsm.br/bitstream/handle/1/15824/Lic_Computacao_MetodologiaPesquisa-Cientifica.pdf?sequence=

Peres, H. H., \& Kurcgant, P. (2004). O ser docente de enfermagem frente a informática [Being a nursing teacher in an informatized world]. Revista latinoamericana de enfermagem, 12(1), 101-108. https://doi.org/10.1590/s0104-11692004000100014

Ramos, C. F. V., Araruna, R. da C., Lima, C. M. F.de, S., Carmen L. A. \& Tanaka, L. H.(2018). Práticas educativas: pesquisa-ação com enfermeiros da Estratégia de Saúde da Família. Revista Brasileira de Enfermagem, 71(3), 1144-1151. https://doi.org/10.1590/0034-7167-2017-0284

Silva, A. C., Bernardes, A., Évora, Y. D., Dalri, M. C., Silva, A. R., \& Sampaio, C. S. (2016). Development of a virtual learning environment for cardiorespiratory arrest training. Revista da Escola de Enfermagem da U S P, 50(6), 990-997. https://doi.org/10.1590/S0080-623420160000700016 